\title{
A High-throughput Targeted Proteomic Approach for Comprehensive Profiling of Methylglyoxal-induced Perturbations of the Human Kinome
}

\author{
Weili Miao*, Yongsheng Xiao*, Lei Guo ${ }^{\dagger}$, Xiaogang Jiang ${ }^{\star}$, Ming Huang $^{\dagger}$, and Yinsheng \\ Wang ${ }^{*}, \dagger$ \\ "Department of Chemistry, University of California, Riverside, CA, 92521-0403, USA \\ †Environmental Toxicology Graduate Program, University of California, Riverside, CA, \\ 92521-0403, USA
}

\section{Abstract}

Kinases are one of the most important families of enzymes that are involved in numerous cell signaling processes. Existing methods for studying kinase expression and activation have limited kinome coverage. Herein, we established a multiple-reaction monitoring (MRM)-based targeted proteomic method that provided an unprecedented coverage $(\sim 80 \%)$ of the human kinome. We employed this method for profiling comprehensively the alterations of the global kinome of HEK293T human embryonic kidney cells upon treatment with methylglyoxal, a glycolysis byproduct that is present at elevated levels in blood and tissues of diabetic patients and is thought to contribute to diabetic complications. Our results led to the quantification of 328 unique kinases. In particular, we found that methylglyoxal treatment gave rise to altered expression of a number of kinases in the MAPK pathway and diminished expression of several receptor tyrosine kinases, including epidermal growth factor receptor (EGFR), insulin growth factor 2 receptor (IGF2R), fibroblast growth factor receptor (FGFR), etc. Furthermore, we demonstrated that the diminished expression of EGFR occurred through a mechanism that is distinct from the reduced expression of IGF2R and FGFR1. Together, our targeted kinome profiling method offers a powerful resource for exploring kinase-mediated signaling pathways that are altered by extracellular stimuli, and the results from the present study suggest new mechanisms underlying the development of diabetic complications.

\section{Graphical Abstract}

Correspondence author: Yinsheng Wang. Telephone: (951)827-2700; yinsheng.wang@ucr.edu.

Supporting Information Available: Details of materials and methods, Table S1 (Sequences for qRT-PCR primers), Table S2 (List of kinases included in kinome library), Table S3 (iRT comparison between desthiobiotin-labeled and desthiobiotin-C3-labeled peptides), Table S4 (List of kinases quantified in 5 replicates and their ratios in MG treated over untreated HEK293T cells), and Figures S1-S5.

This material is available free of charge via the Internet at http://pubs.acs.org. 


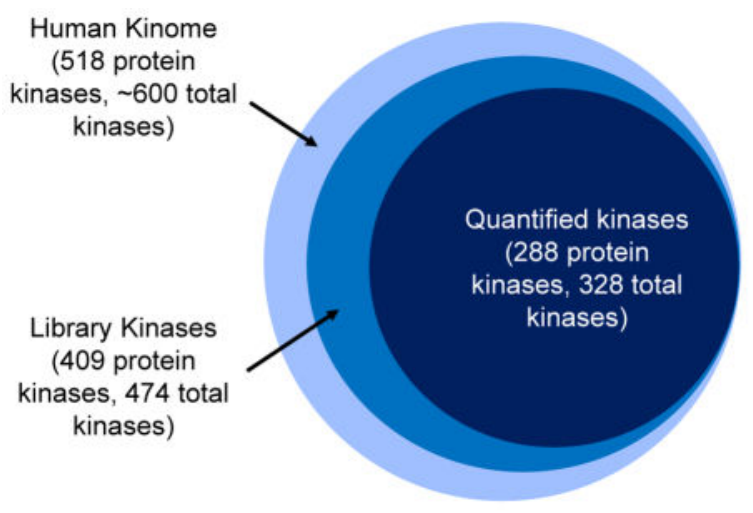

\section{Keywords}

Quantitative proteomics; kinases; methylgloxal; diabetic complications

\section{Introduction}

Kinases are responsible for catalyzing the phosphorylation of numerous biological molecules, ${ }^{1}$ and they play crucial roles in cell signaling and regulating cell proliferation and metabolism. ${ }^{2}$ Aberrant expression and/or activation of kinases have been found in cancer and other human diseases. ${ }^{3}$ However, the extremely low levels of expression of many kinases hamper global kinome studies. Recently published results showed that ATP-affinity probes could be employed for the covalent labeling, enrichment, and subsequent mass spectrometric identification and quantification of kinases with excellent specificity and sensitivity (Figure 1, Figure S1).,5

Targeted proteomics technique, which relies on multiple-reaction monitoring (MRM) on a triple-quadrupole mass spectrometer, has become extensively employed in quantitative proteomics studies. ${ }^{6}$ Compared to data-dependent analysis (DDA)-based discovery mode, the MRM-based targeted proteomic method exhibits a sensitivity that is two orders of magnitude higher towards peptide detection. ${ }^{7}$ We recently developed an MRM-based method for high-throughput assessment of kinase expression and activation. ${ }^{8}$ The previous version of the kinome library encompassed 320 unique peptides from 242 kinases, among which approximately 200 were protein kinases. Importantly, this library contains information about the integrated retention times (iRT) ${ }^{9}$ and unique MRM transitions for kinase peptides. Along with the use of desthiobiotin-containing isotope-coded ATP-affinity probe (ICAP) or stable isotope labeling by amino acids in cell culture (SILAC), the method enables quantitative profiling of the global kinome. ${ }^{10,11}$ The previous version of the library, however, only encompasses approximately $40 \%$ of the human kinome, which leaves a large portion of kinases inaccessible. ${ }^{12}$ In addition, a similar number of kinases were identified with the use of kinome beads for kinase enrichment. ${ }^{13-16}$ Thus, monitoring the majority of the human kinome necessitates the expansion of the MRM kinome library.

Methylglyoxal (MG) is formed from non-enzymatic and enzymatic degradation of triose phosphates produced in the glycolysis pathway (i.e. dihydroxyacetone phosphate and 
glyceraldehyde-3-phosphate), and from the oxidation of hydroxyacetone and aminoacetone. ${ }^{17}$ As a result, hyperglycemia leads to elevated formation of MG, where the serum concentrations of MG increase by 5-6- and 2-3-fold in patients with type- 1 and type-2 diabetes mellitus, respectively. ${ }^{18}$ As a highly reactive a-ketoaldehyde, MG reacts with arginine, lysine and cysteine residues in proteins to yield advanced glycation endproducts (AGEs), and AGE formation is considered a potential link between hyperglycemia and chronic diabetic complications. ${ }^{17}$ In addition, MG can react with DNA and induce the formation of $N^{2}$-(1-carboxyethyl)-2' ${ }^{\prime}$-deoxyguanosine ( $\left.N^{2}-\mathrm{CEdG}\right)$ as the major stable DNA adduct. ${ }^{19,20}$ However, no study has been conducted about how MG perturbs cell signaling by altering the expression or activation of kinases at the global kinome level.

In this study, we expanded substantially the MRM-based kinome library by interrogating the global kinomes of 9 additional human cell lines and by using synthetic desthiobiotinylated kinase peptides. We then employed an MRM-based targeted proteomic method with the expanded kinome library to profile the perturbations of kinome of HEK293T cells upon treatment with methylglyoxal. More than 300 unique kinases were quantified; a number of kinases in the MAPK pathway and several receptor tyrosine kinases were found to be substantially altered in response to methylglyoxal treatment. Together, we expanded successfully our MRM kinome library to achieve an unprecedented coverage of the human kinome and our study also provided important insights into the roles of kinase signaling in the development of diabetic complications.

\section{Research Design and Methods}

\section{Cell culture}

MCF-7 and DU-145 cells (ATCC) were cultured in Eagle's Minimum Essential Medium. GM-00637, GM-04429 (obtained from Prof. Gerd P. Pfeifer), GM15876A (provided by Prof. Karlene Cimprich) ${ }^{21}$, HCT-116, U2OS, and HEK293T (ATCC) cells were cultured in Dulbecco's Modified Eagle Medium. CEM cells (ATCC) were cultured in RPMI 1640 Medium. All cell culture media were supplemented with $10 \%$ fetal bovine serum (Invitrogen, Carlsbad, CA) and penicillin $(100 \mathrm{IU} / \mathrm{mL})$. The cells were maintained at $37^{\circ} \mathrm{C}$ in a humidified atmosphere containing $5 \% \mathrm{CO}_{2}$. Approximately $2 \times 10^{7}$ cells were harvested, washed with cold PBS for three times, and lysed in a 1-mL lysis buffer, which contained $0.7 \%$ CHAPS, $50 \mathrm{mM}$ HEPES (pH 7.4), $0.5 \mathrm{mM}$ EDTA, $100 \mathrm{mM} \mathrm{NaCl}$, and $10 \mu \mathrm{L}$ protease inhibitor cocktail on ice for $30 \mathrm{~min}$. The cell lysates were centrifuged at $16,000 \mathrm{~g}$ at $4{ }^{\circ} \mathrm{C}$ for $30 \mathrm{~min}$ and the resulting supernatants collected.

\section{LC-MS/MS analysis on an LTQ-Orbitrap Velos mass spectrometer}

Endogenous nucleotide removal, ATP-affinity probe labeling, tryptic digestion and affinity purification of desthiobiotin-C3-labeled peptides were performed as previously described. ${ }^{8}$ The resulting peptide mixture was subjected to on-line 2D-LC-MS/MS analysis following previously published procedures ${ }^{8}$ with minor changes (see Supplementary Materials). Synthetic kinase peptides harboring a desthiobiotin-modified lysine were obtained from New England Peptide, Inc. and analyzed by 1D-LC-MS/MS, as described in detail in the Supplementary Materials. 


\section{MRM spectral library construction and LC-MRM analysis}

All LC-MS/MS data obtained from discovery-mode proteomic analysis were searched against the human IPI protein database version 3.68 (87,062 entries) by using MaxQuant (Version 1.3.0.5). ${ }^{22}$ Skyline (version 3.5) ${ }^{23}$ was used to construct spectral libraries for kinase peptides. The same criteria were used to generate peptide list and calculate iRT scores as reported previously, ${ }^{8}$ and details are again provided in the Supplementary Materials.

For monitoring the effect of MG on the alteration of the global kinome in HEK293T cells, the cells were either untreated or treated with $200 \mu \mathrm{M}$ MG for $24 \mathrm{hr}$. After removal of endogenous nucleotides, the lysates of MG-treated cells and control cells were treated with the light and heavy desthiobiotin-C3-ATP probes, respectively, in the forward labeling experiments. The reverse labeling experiments were conducted in the opposite way. A total of three forward and two reverse labeling experiments were conducted. All LC-MRM experiments were conducted on a TSQ Vantage triple-quadrupole mass spectrometer equipped with a nanoelectrospray ionization source coupled to an EASY-nLC II system (Thermo Scientific), as described in detail in the Supplementary Materials.

\section{Western blot}

HEK293T cells were cultured in $75 \mathrm{~cm}^{2}$ cell culture flasks, and the cells were then treated with the indicated concentrations of MG when they reached 40-50\% confluency. The cells were subsequently lysed following the above-described procedures, and $10 \mu \mathrm{g}$ whole cell lysate samples were used for Western blot analysis (see Supplementary Materials).

Phospho-FRS2-a (Tyr196) antibody (Cell Signaling \# 3864, with a 1:1000 dilution), Phospho-FGFR (Tyr653/654) antibody (Cell Signaling \#3471, with a 1:1000 dilution), EGFR 1005 antibody (Santa Cruz Biotechnology, sc-03, with a 1:10000 dilution), IGF2R H-20 antibody (Santa Cruz Biotechnology, sc-14408, with a dilution ratio of 1:1000), and FRS2 H-91 antibody (Santa Cruz Biotechnology, sc-14408, with a dilution ratio of 1:1000) were employed as primary antibodies. Horseradish peroxidase-conjugated anti-rabbit $\operatorname{IgG}$ and Alexa Fluor ${ }^{\circledR} 647$ donkey anti-goat $\operatorname{IgG}(\mathrm{H}+\mathrm{L})$ were used as secondary antibodies. Membranes were also probed with anti-actin antibody (Cell Signaling \#4967, at a dilution ratio of 1:10000) to confirm equal protein loading. Cells were treated with $100 \mathrm{ng} / \mathrm{mL}$ FGF21 (Sigma-Aldrich) for 20 min before harvesting for Western blot analysis of phosphoFRS2- $\alpha$ and phospho-FGFR.

\section{Real-time PCR}

HEK293T cells were seeded in 12-well plates at 50\% confluence level. Total RNA was extracted from cells using TRI Reagent (Sigma). cDNA was synthesized by using M-MLV reverse transcriptase (Promega) and an oligo(dT) ${ }_{16}$ primer. Relative quantification of gene expression was conducted by using qRT-PCR on a Bio-Rad iCycler system (Bio-Rad). The experiment was performed with the use of iQ SYBR Green Supermix Kit (Bio-Rad) and the qRT-PCR primers are listed in Table S1 (see Supplementary Materials). 


\section{Results}

The major objectives of the present study are to expand the MRM kinome library for encompassing the majority of the human kinome and to employ the MRM-based targeted proteomic approach to explore the kinase-mediated signaling events that are perturbed by MG.

\section{Development of an MRM Assay for Human Kinome Profiling}

Expansion of MRM Kinome Library-We first purified, by using avidin agarose beads, the desthiobiotin-C3-labeled kinase peptides from the tryptic digestion mixtures of lysates of 9 human cell lines (i.e. GM-00637, GM-04429, GM15876A, HCT-116, U2OS, CEM, DU-145, HEK293T and MCF-7) that were labeled with the light desthiobiotin-C3-ATP probe (Figure 1a). The resulting desthiobiotin-C3-labeled peptides were analyzed on an LTQ Orbitrap Velos mass spectrometer in the DDA mode, which included a total of more than 200 LC-MS/MS runs. The tandem mass spectra and the retention time information of all desthiobiotin-C3-labeled kinase peptides were imported into Skyline to expand the initial kinome library that was constructed from LC-MS/MS data acquired from 6 human cell lines (i.e. K562, IMR-90, HeLa-S3, Jurkat-T, WM-115, and WM-266-4). ${ }^{8}$ A maximum of five peptides were selected for each kinase, and the three or four most abundant y-ions observed in MS/MS acquired in the DDA mode were employed for MRM monitoring of each peptide.

Peptide selection-Owing to its relatively high reactivity, the desthiobiotin-C3-ATP probe may react with, apart from the lysine residue(s) located at the ATP-binding site of ATP-binding proteins, lysine residues in other proteins through electrostatic interactions. ${ }^{4,5}$ Thus, we classified the kinase peptides from the 15 cell lines in our library into two groups on the basis of local amino acid sequences surrounding the probe-labeled lysine, as described previously. ${ }^{8}$ The first group of peptides harbor at least one of the three ATPbinding motifs (HRDXKXXN, VAXK or GXXXXGK, with ' $\mathrm{X}$ ' being any amino acid), ${ }^{4,5}$ whereas the second group includes those peptides that do not contain any of the three conserved motifs but were identified at least five times in our shotgun proteomics experiments. Although the latter group of peptides do not reside in any of the known conserved ATP-binding motifs and their involvement in ATP binding remains to be determined, their high frequencies of identification in shotgun proteomic studies render these peptides suitable candidates for quantifying the corresponding kinases.

With the use of the ICAP reagents (Figure 1a), a large portion of protein kinases were labeled on the lysine residues located in the ATP-binding motifs that are highly conserved. As a result, some of the identified desthiobiotin-C3-labeled peptides may be assigned to multiple protein kinases. To avoid ambiguity in kinase quantification with the MRM method, we inspected each identified desthiobiotin-C3-labeled peptide in the MRM kinome library and deleted those that could be attributed to multiple kinases.

Synthetic peptides-After combining all unique kinase peptides from the 15 cell lines, the MRM library contains 640 distinct peptides derived from 370 kinases (by using DAVID Bioinformatics Resources), ${ }^{24,25}$ which cover about $60 \%$ of the human kinome. To further expand the library, we checked the sequences of the protein kinases ${ }^{12}$ that were not 
identified from the aforementioned shotgun proteomic analyses. We then designed 192 unique desthiobiotin-labeled peptides that encompass at least one ATP-binding motif of 163 protein kinases, and these peptides were custom-synthesized. The synthetic peptides were then analyzed by LC-MS and MS/MS in the DDA mode, and the acquired data were processed by MaxQuant and incorporated into the Skyline library, as described above. By doing so, we substantially expanded our MRM library to contain 818 unique peptides from 474 distinct human kinases. These include 409 protein kinases, 14 lipid kinases, and 51 other kinases (e.g. nucleoside kinases and carbohydrate kinases, Table S2). Thus, our expanded MRM kinome library has a coverage of approximately $80 \%$ of the human kinome which contains a total of 518 protein kinases. ${ }^{12}$

Retention time calibration-Considering that at least three transitions are needed for each of light- and heavy-probe-labeled peptide, quantitative measurements of 818 kinase peptides by MRM necessitates more than 5000 MRM transitions. To achieve this level of multiplexed detection, it is essential to employ scheduled MRM analysis where the mass spectrometer is programmed to detect only a limited number of peptides in each pre-defined retention time window. Therefore, accurate retention time (RT) prediction for the kinase peptides is essential for our multiplexed MRM-based kinome assay. To this end, we calculated the iRT value for each peptide on our target list following a previously published method. ${ }^{9}$ Based on retention time information of targeted peptides analyzed on an LTQ Orbitrap Velos coupled with EASY-nLC II system, we used 10 BSA peptides as standards and successfully converted empirically determined retention times of $98 \%$ (803 out of 818) kinase peptides into normalized iRT scores, which reflect their conserved elution order. Since the iRT value represents an inherent attribute of the hydrophobicity of a peptide, it can also serve as another parameter to validate the quantification results from MRM assay, where any significant deviation from the linear plot of iRT versus the measured RT could be attributed to false-positive detection.

It is worth noting that the custom-synthesized peptides carry a desthiobiotin-labeled lysine, whereas the kinase peptides arising from ATP affinity probe labeling contain a desthiobiotinC3-labeled lysine. Peptides harboring these two types of modified lysine may display different retention times in LC; thus, it is important to establish the relationship between iRT values for peptides carrying desthiobiotin and desthiobiotin-C3 modifications. To this end, we conducted another set of pull-down experiments with the use of desthiobiotin-ATP probe, which led to the identification of approximately 1000 desthiobiotin-labeled peptides. With the iRT values of the desthiobiotin-labeled kinase peptides and the existing iRT values of the corresponding desthiobiotin-C3-labeled kinase peptides in the library, a linear relationship in iRT values between the desthiobiotin-labeled peptides and the desthiobiotinC3-labeled counterparts could be established (Figure S2a, Table S3). By employing this linear equation and the iRT values for all the desthiobiotin-labeled synthetic peptides, the iRT values for the corresponding desthiobiotin-C3-labeled peptides could be determined and integrated into the MRM library.

Library test-With the above MRM-based assay design, we set out to assess the kinome coverage of the method using the lysate of GM00637 cells. It turned out that the MRM- 
based kinome analysis led to the detection of 470 unique peptides from 313 distinct human kinases in 5 LC-MS/MS runs. On the other hand, the shotgun proteomic approach using online 2D-LC-MS/MS with a 9-step gradient during SCX fractionation only gave rise to the identification of 174 kinases. This result demonstrates that the MRM-based kinome assay provides superior coverage of the human kinome over the DDA method (Figure S2b).

\section{Methylglyoxal-induced alterations of human kinome in HEK293T cells}

We applied the MRM-based kinome library, along with our ICAP probes, to assess the perturbations of the kinome of HEK293T cells upon a 24-hr treatment with $200 \mu \mathrm{M}$ methylglyoxal (Figure S1). In this vein, it is of note that the estimated rate of MG formation in tissues of healthy human subjects was $125 \mu \mathrm{M}$ per day. ${ }^{26}$ In addition, previous studies showed that only about $2-3 \%$ of $M G$ in the culture medium is incorporated into mammalian cells. ${ }^{27,28}$ Thus, the concentration of the MG used in the present study is physiologically relevant.

The results from the MRM-based targeted proteomic method led to the quantification of a total of 328 unique kinases, including 288 protein kinases, 11 lipid kinases and 29 other kinases, which cover approximately 55\% of the human kinome (Figure 2 and Table S4). In the viewpoint that approximately 400 protein kinases are expressed in a single cell, ${ }^{14}$ more than $70 \%$ of the expressed protein kinases could be quantified with the MRM-based kinome assay. All the quantified kinase peptides exhibit an excellent linear fit between the observed retention time and the iRT in the library (Figure 3a). Similar relative abundances of 3 transitions obtained from MRM and shotgun proteomic analyses illustrate the robust quantification of the kinase peptides by MRM analysis (Figure 3c, d). More than $90 \%$ of the quantified kinase peptides displayed a consistent trend in forward and reverse labeling experiments in MRM-based targeted analysis (Table S4, and representative results for EGFR are shown in Figure 3b), demonstrating the excellent performance of this method.

Among the 328 quantified kinases, 40 and 9 were down- and up-regulated (with at least 1.5fold change), respectively, in HEK293T cells following a 24-hr treatment with $200 \mu \mathrm{M}$ MG. In addition, 11 out of 49 perturbed kinases are involved in MAPK pathway, rendering it the major pathway perturbed by MG treatment (Figure S3, Figure 4a). In this vein, the MGinduced alteration of MAPK signaling pathway was reported previously. ${ }^{29,30}$ Furthermore, 8 receptor tyrosine kinases (RTKs), including EPHA2, EPHA4, EGFR, IGF2R, FGFR1, FGFR2, FGFR3 and NTRK2, were found to be altered upon MG treatment and all of them except FGFR2 were down-regulated (Figure 4b).

\section{MG led to the down-regulation of RTKs}

EGFR, IGF2R, and FGFR1 were found to be related to diabetes ${ }^{31-33}$ and were all revealed by our global kinome profiling data to be down-regulated upon MG treatment (Figure S4, Figure $4 \mathrm{~b}$ ). Thus, we focused on these kinases to explore the mechanisms through which their alterations are induced by MG treatment. To this end, we first examined whether the diminished levels of EGFR, IGF2R and FGFR1 proteins are due to reduced mRNA expression of the corresponding genes. Our real-time PCR results showed that only the 
mRNA level of EGFR gene was decreased with the dose of MG, whereas no apparent change in mRNA level was observed for IGF2R or FGFR gene (Figure 5a).

We next validated the MRM findings by using Western blot analysis and assessed whether the MG-induced decrease of the three receptor tyrosine kinases could be rescued by preincubation of cells with scavengers of a-ketoaldehydes and an antioxidant. Indeed our Western blot results revealed that EGFR protein displayed a time- and dose-dependent decrease in expression level in HEK293T cells upon MG treatment (Figure 5b\&c).

Aminoguanidine (AG) and metformin (MET) are scavengers of a-ketoaldehydes, and thus they inhibit the formation of MG-induced advanced glycation end-products. ${ }^{34,35} \mathrm{~N}$-acetyl- $L$ cysteine (NAC), as an antioxidant, inhibits ROS-dependent apoptosis. ${ }^{36}$ To exploit further the mechanisms underlying the MG-induced decline in EGFR protein level, we pretreated HEK293T cells with $1 \mathrm{mM} \mathrm{AG,} \mathrm{NAC} \mathrm{or} \mathrm{MET} \mathrm{for} 7 \mathrm{hr}$ prior to a 24-hr treatment with 500 $\mu \mathrm{M}$ MG. Our results showed that the pretreatment with NAC, but not AG or MET, could significantly restore the expression level of EGFR (Figure 5d).

In line with the MRM data, Western blot result also revealed a dose-dependent decrease in IGF2R protein level in HEK293T cells upon MG treatment (Figure 6a). In addition, the MGinduced decrease in IGF2R expression could be rescued by pretreatment of HEK293T cells with NAC, AG or MET (Figure 6b). FGFR activation with the use of recombinant fibroblast growth factor 21 has been proposed for the treatment of diabetes, ${ }^{37}$ and our MRM kinome assay revealed the diminished expression/activation of FGFR1 upon MG treatment. Because of the relatively low level of expression of FGFR1 in HEK293T cells, we were not able to detect FGFR1 protein in these cells using Western blot (data not shown). Instead, we employed Western blot to monitor the effects of MG treatment on FGF21-mediated FGFR1 activation by assessing its autophosphorylation on Tyr653/654 38 and the phosphorylation of its substrate protein, FRS2. ${ }^{39}$ Indeed we found that MG treatment resulted in diminished phosphorylation of FGFR1 and FRS2. Moreover, the reduced phosphorylation of these two proteins could again be rescued by preincubation of HEK293T cells with NAC, AG or MET (Figure S5). Together, the above results suggest that MG elicited the decreased levels of FGFR1 and IGF2R through a common mechanism, which differs from that underlying the MG-induced down-regulation of EGFR.

\section{Discussion}

Reversible phosphorylation of proteins and other biomolecules by kinases and phosphatases constitute one of the most important and best studied pathways in cell signaling. Thus, highthroughput methods for global profiling of the human kinome will enable systematic interrogation of kinase-mediated molecular events conferred by intracellular signaling and extracellular cues. However, existing methods for profiling the global human kinome are hampered by the limited kinome coverage. In the present study, we made significant advances toward this challenge by establishing an MRM-based targeted proteomic approach with an unprecedented coverage of the human kinome. In particular, our expanded kinome library covers approximately $80 \%$ of human kinome and contains 474 human kinases (488 unique IPIs), among which 409, 14 and 51 were protein kinases, lipid kinases, and other kinases, respectively. 
This method was successfully employed to assess the alterations of the kinome in HEK293T cells after MG treatment, which allowed for the quantification of 328 kinases. Among these quantified kinases, 49 were perturbed, 8 of which were RTKs. Further experiments on selected receptor tyrosine kinases showed that MG led to a dose-dependent decrease in the level of EGFR protein via a mechanism that differs from the diminished expression of IGF2R and FGFR1 proteins. While MG treatment led to a dose-dependent decline in mRNA level of EGFR gene, the mRNA expression of IGF2R or FGFR1 gene was not perturbed by MG exposure. In addition, while the MG-triggered decrease in all three receptor tyrosine kinases could be restored by preincubation of cells with an antioxidant (i.e. NAC), scavengers of a-ketoaldehydes (i.e. AG and MET) could only rescue the MG-induced reduction of IGF2R and FGFR1 proteins, but not that of EGFR. The lack of effect of AG or MET on restoring the MG-elicited decrease in EGFR protein expression suggests that MG may bind directly to some cell-surface proteins, which may stimulate the production of ROS and ultimately lead to transcriptional down-regulation of EGFR gene. The detailed molecular events underlying these processes, however, await further investigation. In addition, the capability of NAC in restoring the MG-induced decreases in all three receptor tyrosine kinases is in keeping with the previous proposal that hyperglycemia-induced ROS activates many pathways of tissue damage in diabetic patients. ${ }^{40,41}$ Our results suggest that the inhibition of RTKs may contribute to the development of diabetic complications, and restoration of the functions of RTKs may provide new venues for the therapeutic interventions of diabetic complications.

\section{Supplementary Material}

Refer to Web version on PubMed Central for supplementary material.

\section{Acknowledgments}

This work was supported by the National Institute of Health (R01 DK082779).

\section{References}

1. Cheng HC, Qi RZ, Paudel H, Zhu HJ. Enzyme Res. 2011; 2011:794089. [PubMed: 22195276]

2. Lemmon MA, Schlessinger J. Cell. 2010; 141:1117-1134. [PubMed: 20602996]

3. Blume-Jensen P, Hunter T. Nature. 2001; 411:355-365. [PubMed: 11357143]

4. Xiao Y, Guo L, Jiang X, Wang Y. Anal Chem. 2013; 85:3198-3206. [PubMed: 23413923]

5. Xiao Y, Guo L, Wang Y. Anal Chem. 2013; 85:7478-7486. [PubMed: 23841533]

6. Picotti P, Aebersold R. Nat Meth. 2012; 9:555-566.

7. Lange V, Picotti P, Domon B, Aebersold R. Mol Syst Biol. 2008; 4:222. [PubMed: 18854821]

8. Xiao Y, Guo L, Wang Y. Mol Cell Proteomics. 2014; 13:1065-1075. [PubMed: 24520089]

9. Escher C, Reiter L, MacLean B, Ossola R, Herzog F, Chilton J, MacCoss MJ, Rinner O. Proteomics. 2012; 12:1111-1121. [PubMed: 22577012]

10. Guo L, Xiao Y, Fan M, Li JJ, Wang Y. J Proteome Res. 2015; 14:193-201. [PubMed: 25341124]

11. Guo L, Xiao Y, Wang Y. Anal Chem. 2014; 86:10700-10707. [PubMed: 25301106]

12. Manning G, Whyte DB, Martinez R, Hunter T, Sudarsanam S. Science. 2002; 298:1912-1934. [PubMed: 12471243]

13. Stuhlmiller TJ, Miller SM, Zawistowski JS, Nakamura K, Beltran AS, Duncan JS, Angus SP, Collins KA, Granger DA, Reuther RA, Graves LM, Gomez SM, Kuan PF, Parker JS, Chen X, 
Sciaky N, Carey LA, Earp HS, Jin J, Johnson GL. Cell Rep. 2015; 11:390-404. [PubMed: 25865888]

14. Duncan JS, Whittle MC, Nakamura K, Abell AN, Midland AA, Zawistowski JS, Johnson NL, Granger DA, Jordan NV, Darr DB, Usary J, Kuan PF, Smalley DM, Major B, He X, Hoadley KA, Zhou B, Sharpless NE, Perou CM, Kim WY, Gomez SM, Chen X, Jin J, Frye SV, Earp HS, Graves LM, Johnson GL. Cell. 2012; 149:307-321. [PubMed: 22500798]

15. Daub H, Olsen JV, Bairlein M, Gnad F, Oppermann FS, Korner R, Greff Z, Keri G, Stemmann O, Mann M. Mol Cell. 2008; 31:438-448. [PubMed: 18691976]

16. Oppermann FS, Gnad F, Olsen JV, Hornberger R, Greff Z, Kéri G, Mann M, Daub H. Mol Cell Proteomics. 2009; 8:1751-1764. [PubMed: 19369195]

17. Thornalley PJ. Gen Pharmacol. 1996; 27:565-573. [PubMed: 8853285]

18. McLellan AC, Thornalley PJ, Benn J, Sonksen PH. Clin Sci. 1994; 87:21-29. [PubMed: 8062515]

19. Yuan B, Cao H, Jiang Y, Hong H, Wang Y. Proc Natl Acad Sci U S A. 2008; 105:8679-8684. [PubMed: 18562283]

20. Murata-Kamiya N, Kamiya H. Nucl Acids Res. 2001; 29:3433-3438. [PubMed: 11504881]

21. Bomgarden RD, Lupardus PJ, Soni DV, Yee MC, Ford JM, Cimprich KA. EMBO J. 2006; 25:2605-2614. [PubMed: 16675950]

22. Cox J, Mann M. Nat Biotechnol. 2008; 26:1367-1372. [PubMed: 19029910]

23. MacLean B, Tomazela DM, Shulman N, Chambers M, Finney GL, Frewen B, Kern R, Tabb DL, Liebler DC, MacCoss MJ. Bioinformatics. 2010; 26:966-968. [PubMed: 20147306]

24. Huang DW, Sherman BT, Lempicki RA. Nat Protocols. 2008; 4:44-57.

25. Huang DW, Sherman BT, Lempicki RA. Nucleic Acids Res. 2009; 37:1-13. [PubMed: 19033363]

26. Phillips SA, Thornalley PJ. Eur J Biochem. 1993; 212:101-105. [PubMed: 8444148]

27. Che W, Asahi M, Takahashi M, Kaneto H, Okado A, Higashiyama S, Taniguchi N. J Biol Chem. 1997; 272:18453-18459. [PubMed: 9218489]

28. Riboulet-Chavey A, Pierron A, Durand I, Murdaca J, Giudicelli J, Van Obberghen E. Diabetes. 2006; 55:1289-1299. [PubMed: 16644685]

29. Miyata S, Miyazaki H, Liu B-F, Fukunaga M, Hamada Y, Ueyama S, Muramoto O, Jiang QY, Kusunoki H, Uriuhara A, Hirota Y, Kasuga M. Int Congress Series. 2002; 1245:87-89.

30. Liu B-F, Miyata S, Hirota Y, Higo S, Miyazaki H, Fukunaga M, Hamada Y, Ueyama S, Muramoto O, Uriuhara A, Kasuga M. Kidney Int. 2003; 63:947-957. [PubMed: 12631075]

31. McCann JA, Xu YQ, Frechette R, Guazzarotti L, Polychronakos C. J Clin Endocrinol Metab. 2004; 89:5700-5706. [PubMed: 15531531]

32. Buteau J, Foisy S, Joly E, Prentki M. Diabetes. 2003; 52:124-132. [PubMed: 12502502]

33. Wu A-L, Kolumam G, Stawicki S, Chen Y, Li J, Zavala-Solorio J, Phamluong K, Feng B, Li L, Marsters S, Kates L, van Bruggen N, Leabman M, Wong A, West D, Stern H, Luis E, Kim HS, Yansura D, Peterson AS, Filvaroff E, Wu Y, Sonoda J. Sci Transl Med. 2011; 3:113ra126113 ra126.

34. Thornalley PJ, Yurek-George A, Argirov OK. Biochem Pharmacol. 2000; 60:55-65. [PubMed: 10807945]

35. Ruggiero-Lopez D, Lecomte M, Moinet G, Patereau G, Lagarde M, Wiernsperger N. Biochem Pharmacol. 1999; 58:1765-1773. [PubMed: 10571251]

36. Curtin JF, Donovan M, Cotter TG. J Immunol Meth. 2002; 265:49-72.

37. Kharitonenkov A, Shiyanova TL, Koester A, Ford AM, Micanovic R, Galbreath EJ, Sandusky GE, Hammond LJ, Moyers JS, Owens RA, Gromada J, Brozinick JT, Hawkins ED, Wroblewski VJ, Li DS, Mehrbod F, Jaskunas SR, Shanafelt AB. J Clin Invest. 2005; 115:1627-1635. [PubMed: 15902306]

38. Furdui CM, Lew ED, Schlessinger J, Anderson KS. Mol Cell. 2006; 21:711-717. [PubMed: 16507368]

39. Kouhara H, Hadari YR, Spivak-Kroizman T, Schilling J, Bar-Sagi D, Lax I, Schlessinger J. Cell. 1997; 89:693-702. [PubMed: 9182757]

40. Brownlee M. Nature. 2001; 414:813-820. [PubMed: 11742414] 
41. Brownlee M. Diabetes. 2005; 54:1615-1625. [PubMed: 15919781] 
a<smiles>[X]C([X])([X])C([X])([X])C([X])([X])C(=O)OP(=O)(O)OP(=O)(O)OP(=O)(O)OCC1OC(n2cnc3c(N)ncnc32)C(O)C1O</smiles>

b

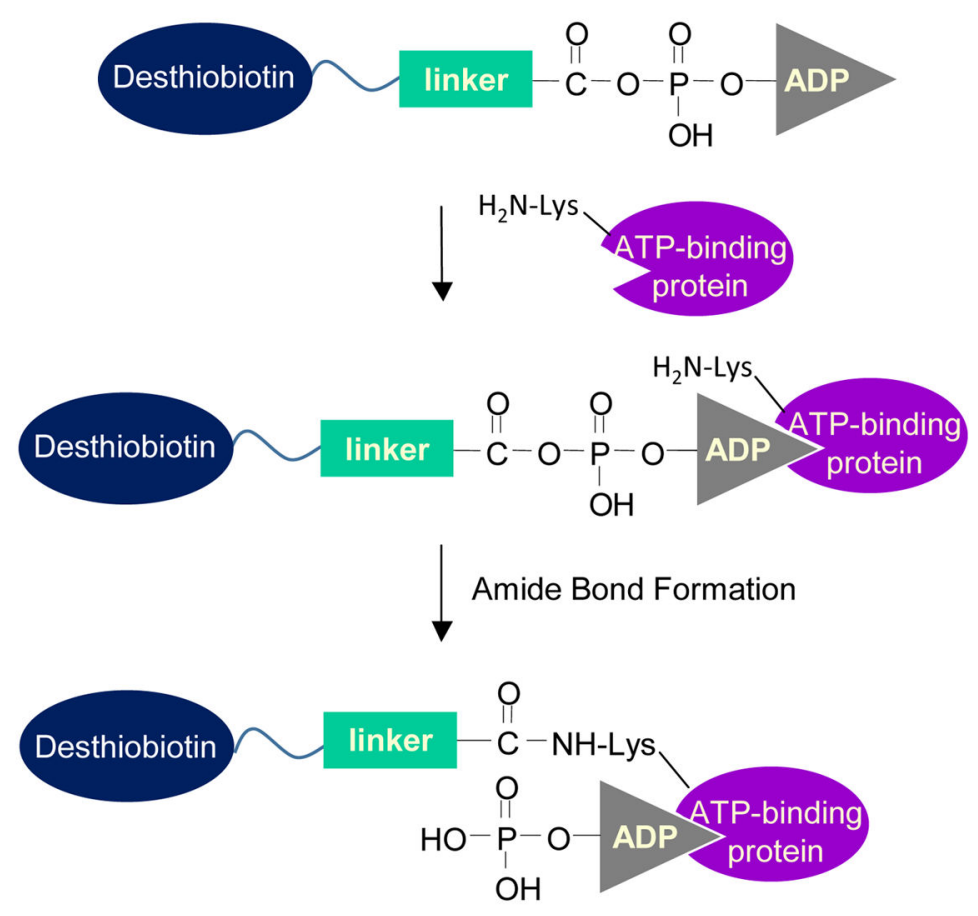

Figure 1.

A targeted proteomic approach for interrogating the human kinome. (a) The chemical structure of the ICAP probe. (b) The working principle of ICAP probe. 

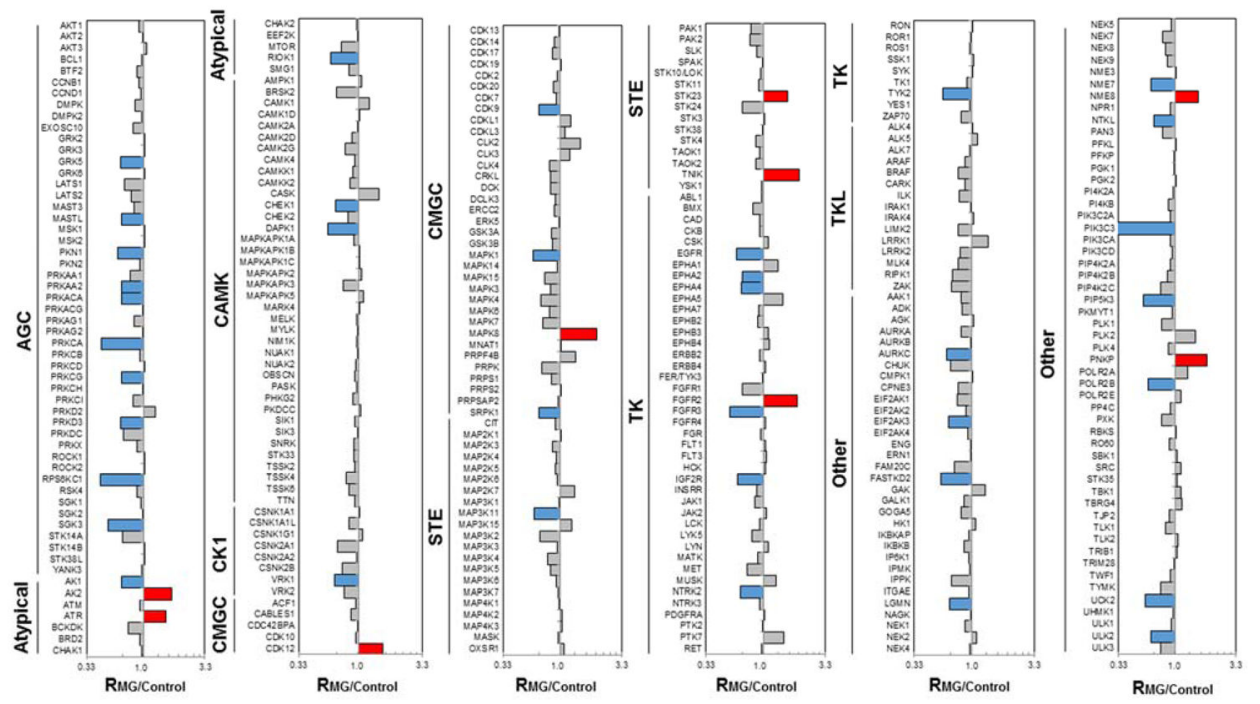

Figure 2.

MG-induced alterations of human kinome in HEK293T cells. 

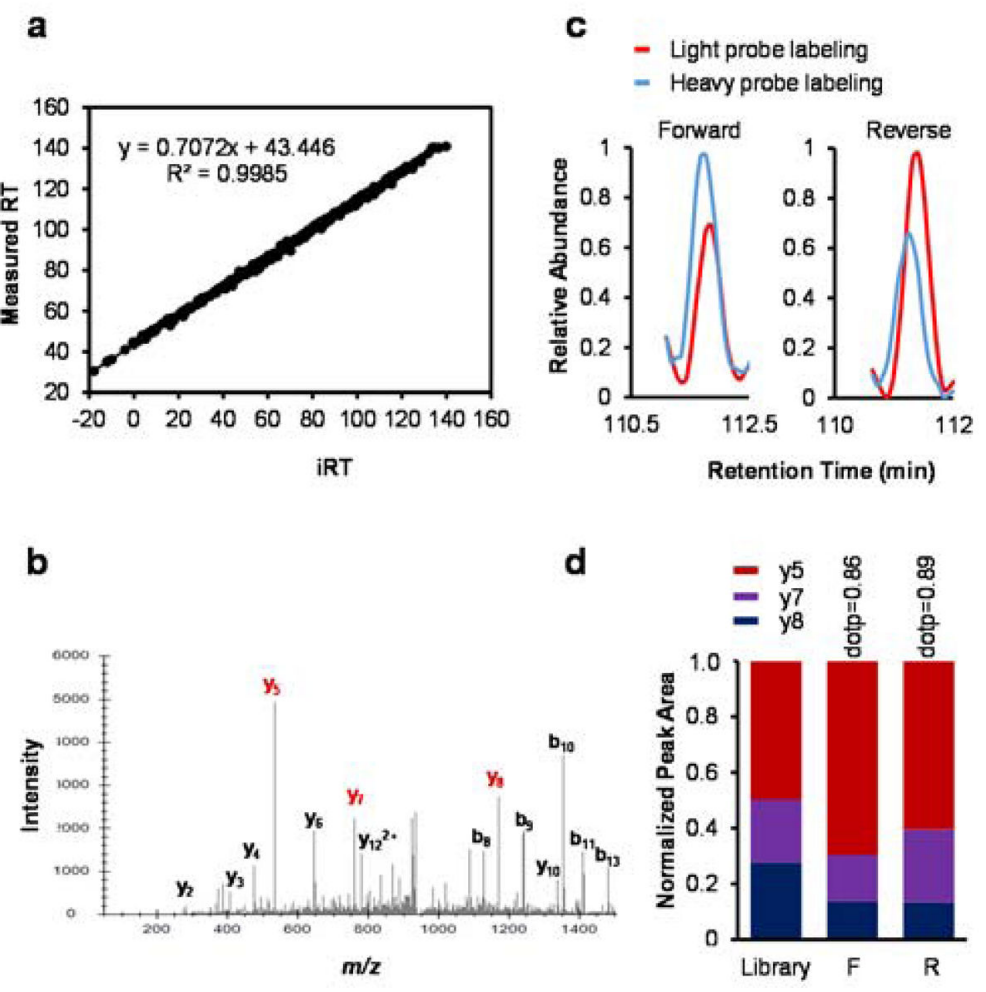

Figure 3.

Representative MRM results for EGFR. (a) Correlation between iRT in library and measured RT on a TSQ Vantage mass spectrometer. (b) MS/MS of a representative peptide ITDFGLAK\#LLGAEEK from EGFR (the $\mathrm{y}_{5}, \mathrm{y}_{7}$ and $\mathrm{y}_{8}$ ions labeled in red represent the 3 transitions used for MRM analysis). (c) MRM traces for peptide ITDFGLAK\#LLGAEEK from EGFR derived from forward and reverse labeling experiments. (d) Relative abundances of three fragment ions monitored in MRM analysis compared to those in MS/MS acquired from shotgun proteomic analysis. 'dotp' represents dot product among relative abundances of 3 fragment ions observed in MRM run and DDA run. ' $F$ ' and ' $R$ ' refer to data obtained from forward and reverse labeling experiments. In forward labeling, the light and heavy desthiobiotin-C3-ATP probes were treated with the lysates of MG-treated and control HEK293T cells, respectively. The opposite was conducted for reverse-labeling experiments. 


$$
\text { a }
$$

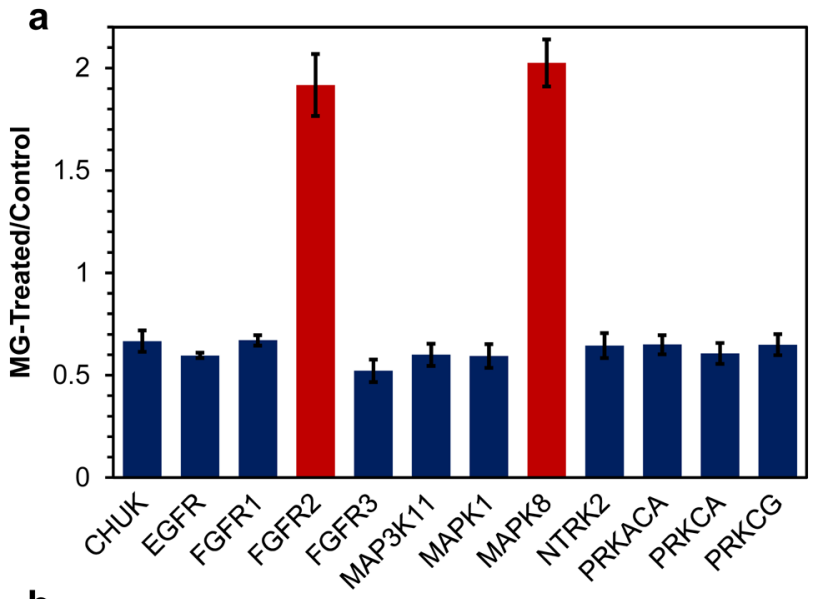

b

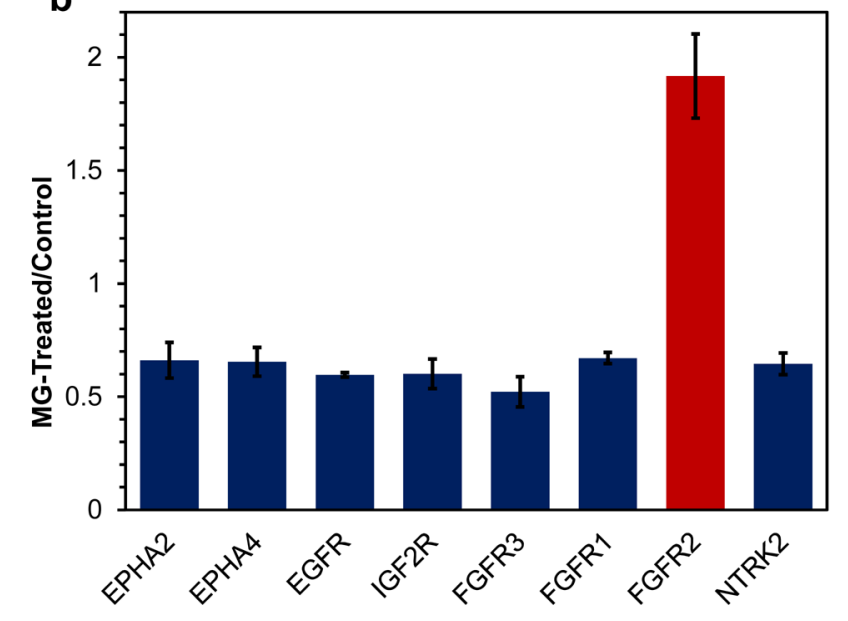

Figure 4.

The quantification results for kinases involved in the MAPK pathway (a) and receptor tyrosine kinases (b) that were altered upon MG treatment. The data represent the mean and standard deviation of results obtained from five labeling experiments, which included three forward and two reverse labeling experiments. In forward labeling, the light and heavy desthiobiotin-C3-ATP probes were treated with the lysates of MG-treated and control HEK293T cells, respectively. The opposite was conducted for reverse labeling experiments. 
b

- Control $=200 \mu \mathrm{M} M G=500 \mu \mathrm{M} M G$

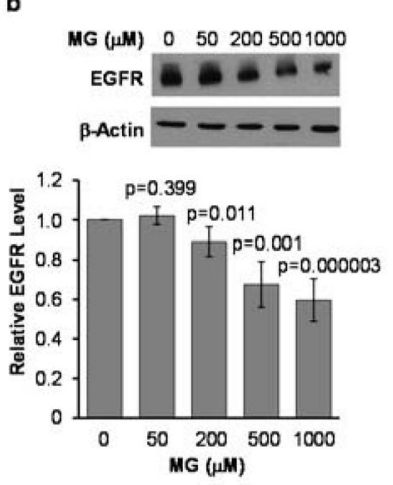

c
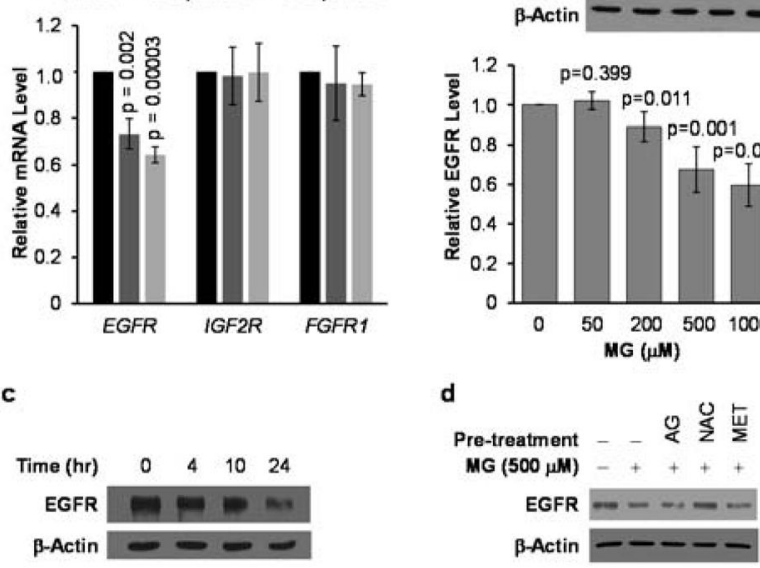

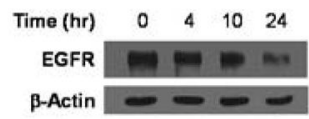
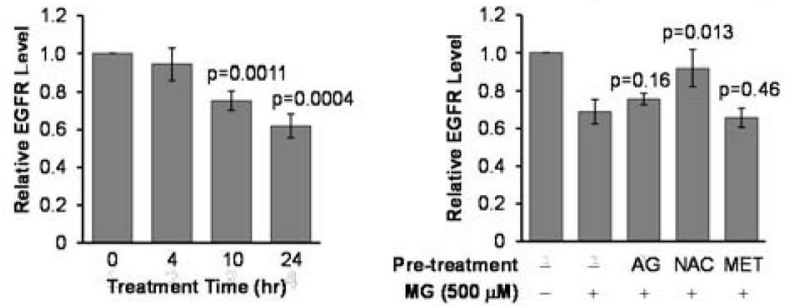

Figure 5.

MG treatment led to diminished level of EGFR protein. (a) Changes in mRNA levels of EGFR, IGF2R and FGFR1 genes after treatment with different doses of $\mathrm{MG}(\mathrm{n}=3)$. (b) Quantification results showing the dose-dependent changes in expression levels of EGFR protein after MG treatment $(n=3)$ and representative Western blot result. (c) Quantification results showing the time-dependent changes in expression levels of EGFR protein after MG treatment $(n=3)$ and representative Western blot result. (d) Quantification results showing that the decrease in expression level of EGFR induced by MG treatment could be rescued by pre-treatment with NAC, but not AG or MET. Shown also are the Western blot result. All p values were calculated by using unpaired, two-tailed $t$-test, and the p-values in (d) referred to the comparisons with the results obtained from cells treated with MG alone. The concentrations of AG, NAC, and MET used for pretreatment were $1 \mathrm{mM}$ each. 
a
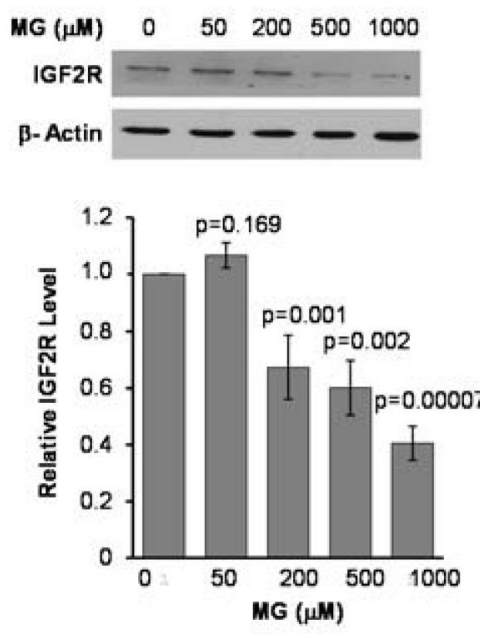

b
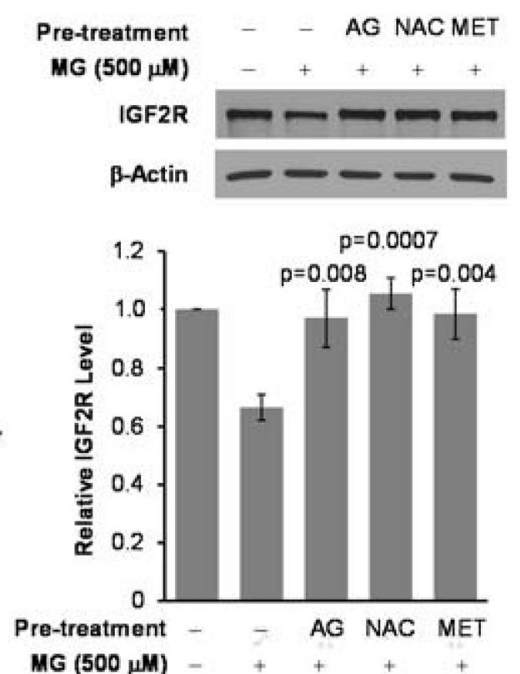

Figure 6.

MG treatment led to diminished level of IGF2R protein. (a) Quantification results showing the dose-dependent alterations in expression levels of IGF2R protein after MG treatment ( $\mathrm{n}$ =3) and representative Western blot result. (b) The decrease in expression level of IGF2R protein induced by MG treatment could be rescued by pre-incubation of cells with NAC, but not AG or MET $(n=3)$. Shown also are the representative Western blot result. All $p$ values were calculated by using unpaired, two-tailed $t$-test, and the p-values in (b) referred to the comparisons with the results obtained from cells treated with $\mathrm{MG}$ alone. The concentrations of AG, NAC, and MET used for pretreatment were $1 \mathrm{mM}$ each. 